\title{
PERTURBATION RESULTS RELATED TO PALINDROMIC EIGENVALUE PROBLEMS
}

\author{
E. K.-W. CHU ${ }^{\otimes 1}$, W.-W. LIN $^{2}$ and C.-S. WANG ${ }^{3}$
}

(Received 13 September, 2007; revised 30 September, 2008)

\begin{abstract}
We investigate the perturbation of the palindromic eigenvalue problem for the matrix quadratic $P(\lambda)=\lambda^{2} A_{1}^{\star}+\lambda A_{0}+A_{1}$ with $A_{0}, A_{1} \in \mathcal{C}^{n \times n}$ and $A_{0}^{\star}=A_{0}$ (where $\star=T$ or $H$ ). The perturbation of eigenvalues in the context of general matrix polynomials, palindromic pencils, (semi-Schur) anti-triangular canonical forms and differentiation is discussed.

2000 Mathematics subject classification: primary 15A18, 15A22, 65F15.

Keywords and phrases: anti-triangular form, eigenvalue, eigenvector, matrix polynomial, palindromic eigenvalue problem, palindromic linearization, palindromic pencil, perturbation.
\end{abstract}

\section{Introduction}

Consider the matrix quadratic

$$
P(\lambda) \equiv \lambda^{2} A_{1}^{\star}+\lambda A_{0}+A_{1},
$$

where $A_{0}, A_{1} \in \mathcal{C}^{n \times n}$ with $A_{0}^{\star}=A_{0}(\star=T$ or $H)$, and the corresponding palindromic quadratic eigenvalue problem

$$
P(\lambda) x=0, \quad x \neq 0
$$

In this paper we consider only regular matrix polynomials $P(\lambda)$, where "regular" is understood to mean $\operatorname{det} P(\lambda) \not \equiv 0$.

\footnotetext{
${ }^{1}$ School of Mathematical Sciences, Building 28, Monash University, VIC 3800, Australia; e-mail: eric.chu@sci.monash.edu.au.

${ }^{2}$ Department of Applied Mathematics, National Chiao Tung University, Hsinchu 300, Taiwan; e-mail:wwlin@am.cthu.edu.tw.

${ }^{3}$ Department of Mathematics, National Cheng Kung University, Tainan 701, Taiwan; e-mail: cswang@math.ncku.edu.tw.

(C) Australian Mathematical Society 2009, Serial-fee code 1446-1811/09 \$16.00
} 
From the transpose or Hermitian of (1.1), a palindromic eigenvalue problem is seen to possess a spectrum $\sigma(P)$ that contains both $\lambda$ and its "reciprocal" $1 / \lambda^{\star}$ (with 0 and $\infty$ considered to be reciprocal to each other). Under favourable conditions, the eigenvalue problem of the original matrix polynomial $P(\lambda)$ has a palindromic linearization of the form $\lambda Z \pm Z^{\star}[6,10,16]$.

We can transform $\lambda Z-Z^{\star}$ to the form $v(-Z)+(-Z)^{\star}$, with $v=-\lambda$. Similarly, $\lambda^{2} A_{1}^{\star}+\lambda A_{0}+A_{1}$ and $v^{2} A_{1}^{\star}-v A_{0}+A_{1}$ define equivalent palindromic eigenvalue problems. As a consequence, and for simplicity of presentation, we shall concentrate on the palindromic eigenvalue problem. Anti-palindromic or odd/even eigenvalue problems [16-18] can be treated similarly.

A solid foundation for the solution of palindromic eigenvalue problems has been laid by Hilliges, D.S. Mackey, N. Mackey, Mehl and Mehrmann (see [13, 16, 17]). An alternative approach to tackling the problem which involves structure-preserving doubling algorithms can be found in [6]. Recently, quadratic eigenvalue problems [21] have attracted much interest. An important example of palindromic eigenvalue problems can be found in the vibration analysis of fast trains; see [13] or [14] for a general introduction and [12] for details. For the general perturbation of eigenvalues for polynomial eigenvalue problems, see [5, 20]; consult also [8, 15] for some related results.

This paper is organized as follows. In Sections 2-5, the perturbation of eigenvalues in terms of general matrix polynomials, palindromic linearizations, the anti-triangular canonical form [16-18] and the semi-Schur anti-triangular canonical form is investigated by means of the Bauer-Fike technique for perturbations of arbitrary size [5]. The derivatives of eigenvalues and eigenvectors of $P(\lambda)$ are considered in Section 6. We summarize our conclusions in Section 7. The results in Section 3-5 are applicable to the more general palindromic pencils $Z-\lambda Z^{\star}$, which may not be linearizations of any matrix polynomials.

Note that Sun and Stewart's implicit function approach [19] has been applied to palindromic linearizations, general matrix quadratics and palindromic eigenvalue problems in [7], to obtain perturbation results for (simple) eigenvalues and the corresponding deflating subspaces.

It is important to distinguish between the different perturbation techniques. Note that the Bauer-Fike technique allows perturbations of arbitrary size, and the clustering of eigenvalues (and hence the corresponding deflating subspaces) may vary greatly. Consequently, it is meaningless to talk about perturbation of eigenvectors or deflating subspaces in the Bauer-Fike-type perturbation theorems of Sections 2-5. Perturbation results obtained via differentiation or implicit function approaches are valid only for asymptotically small perturbations, but results for deflating subspaces are available. Note that only simple eigenvalues (or the sums and averages of multiple eigenvalues) are differentiable, so generalized derivatives (or subgradients) have to be utilized in general [4]. We remark that the naive differentiation technique employed in Section 6 assumes differentiability, which can only be proved rigorously by using tools such as the implicit function theorem. 
Next, we give a few words of warning. Comparison of perturbation results is a risky art. Typically, error bounds and condition numbers are simplified upper bounds of more complicated quantities, and a better (worse) upper bound does not always imply a smaller (bigger) error. Furthermore, optimization of such upper bounds, though often possible, is seldom attempted because of cost or inconvenience, making comparisons of perturbation results even more perilous. Therefore, we do not claim to have found the "best" perturbation results, if such exist at all. We shall quite often interpret perturbation results qualitatively, rather than apply them quantitatively, and we will indicate when things may go wrong or pitfalls to avoid. Nevertheless, our perturbation results, in addition to those in [2, 7, 15], are among the very few that are currently available for palindromic eigenvalue problems, and should be of use in related investigations. Lastly, conditions qualifying when perturbations are large or (asymptotically) small can be written down but are complicated and rarely checked. Again, such perturbation results may have to be used qualitatively rather than quantitatively.

\section{Bauer-Fike theorem for general matrix polynomials}

The unstructured perturbation result for general matrix polynomials, presented in Theorem 2.1 below, may not be directly applicable or satisfactory for palindromic eigenvalue problems. However, it serves as a reference for the structured perturbation results that we consider in later sections. Also, palindromic eigenvalue problems are sometimes perturbed in an unstructured manner; one example is when the QZ algorithm [11] is applied to an associated palindromic linearization $Z-\lambda Z^{\star}$. The associated perturbation problem has to be treated as an unstructured one, using the theorem below.

We now state, without proof, [5, Theorem 4.2] on the perturbation of eigenvalues of a general matrix polynomial.

THEOREM 2.1. Consider a regular matrix polynomial

$$
L(\alpha, \beta) \equiv \sum_{j=0}^{l} B_{j} \alpha^{j} \beta^{l-j}
$$

and its perturbation

$$
\widetilde{L}(\alpha, \beta) \equiv \sum_{j=0}^{l} \widetilde{B}_{j} \alpha^{j} \beta^{l-j}, \quad \widetilde{B}_{j} \equiv B_{j}+\delta B_{j} \quad(j=0, \ldots, l) .
$$

Let $(X, T, Z)$ be a resolvent triple for $L$ (see $[5,10])$ which is constructed using some finite and infinite Jordan pairs, $J_{F}$ and $J_{\infty}$. For $\left(\alpha_{i}, \beta_{i}\right) \in \sigma(L)$ and $(\alpha, \beta)$ $\in \sigma(\widetilde{L})$ with the scaling $\left|\alpha_{i}\right|^{2}+\left|\beta_{i}\right|^{2}=1=|\alpha|^{2}+|\beta|^{2}$, the spectral variation of $\widetilde{L}$ from $L$ is defined as

$$
s_{L}(\widetilde{L}) \equiv \max _{(\alpha, \beta)}\left\{s_{(\alpha, \beta)}\right\}, \quad s_{(\alpha, \beta)} \equiv \min _{i}\left\{\left|\alpha \beta_{i}-\beta \alpha_{i}\right|\right\}
$$


Let $p$ be the maximum dimension of the Jordan blocks in $J_{F}$ or $J_{\infty}$.

Then, for $\|\cdot\|=\|\cdot\|_{\tau}$ (where $\tau=1,2$ or $\left.\infty\right)$,

$$
s_{(\alpha, \beta)} \leq \max \left\{\theta_{1}, \theta_{1}^{1 / p}\right\}, \quad \theta_{1} \equiv p \mathcal{F} \kappa \Delta
$$

where $\mathcal{F} \equiv c_{1} \sqrt{(l+1) / 2}$ with $c_{1}=1($ for $\tau=1,2)$ or $c_{1}=\sqrt{l}($ for $\tau=\infty)$ and

$$
\kappa \equiv\|X\| \cdot\|Z\|, \quad \Delta \equiv\left\|\left[\delta B_{0}, \ldots, \delta B_{l}\right]\right\| .
$$

Also,

$$
s_{L}(\widetilde{L}) \leq \max \left\{\theta_{1}, \theta_{1}^{1 / p}\right\}
$$

COMMENTS 2.2.

(a) Note that, in the above theorem, we use the representation $(\alpha, \beta)$ for $\lambda=\alpha / \beta$.

(b) In the palindromic case we have $l=2, B_{0}=A_{1}^{\star}=B_{2}^{\star}$ and $B_{1}=A_{0}=A_{0}^{\star}$. Ultimately, the perturbation of the palindromic eigenvalues is controlled by $\theta_{1}$ in (2.2), which is in turn dominated by the error term involving $\left\|\left[\delta A_{0}, \delta A_{1}\right]\right\|$. The condition of the eigenvalues will be poor when $\kappa$ is large or when deflating subspaces for different eigenvalues are getting "close" to each other, making the resolution of the spectrum more and more difficult. Note also that the perturbation in $\delta A_{0}$ may be nonsymmetric, pushing a pair of reciprocal palindromic eigenvalues to ones that are not reciprocal (or approximately reciprocal when $\delta A_{0}$ is small). For a symmetric $\delta A_{0}$, we only have to consider the perturbation of half of the eigenvalues, owing to the palindromic structure.

(c) Based on Theorem 2.1, we can consider the perturbation of a cluster of eigenvalues; for details, see [5, Section 5.2]. A cluster, to be defined later in (2.3), can be one simple eigenvalue, a group of multiple eigenvalues or a group of neighbouring eigenvalues. For $(\alpha, \beta) \notin \sigma(L)$, assume the following decomposition of the resolvent:

$$
L(\alpha, \beta)^{-1}=X_{1} T_{1}(\alpha, \beta)^{-1} Z_{1}+X_{2} T_{2}(\alpha, \beta)^{-1} Z_{2},
$$

where $\left(\left[X_{1}, X_{2}\right], T_{1} \oplus T_{2},\left[Z_{1}, Z_{2}\right]\right)$ is a resolvent triple [10] appropriately partitioned into two parts. The eigenvalues in $T_{1}$ form a cluster when

$$
\left\|X_{1} T_{1}(\alpha, \beta)^{-1} Z_{1}\right\| \gg\left\|X_{2} T_{2}(\alpha, \beta)^{-1} Z_{2}\right\| \leq \epsilon\left\|X_{1} T_{1}(\alpha, \beta)^{-1} Z_{1}\right\|
$$

for some small constant $\epsilon$. Consequently,

$$
\left\|L(\alpha, \beta)^{-1}\right\| \leq(1+\epsilon)\left\|X_{1} T_{1}(\alpha, \beta)^{-1} Z_{1}\right\| .
$$

Arguments and techniques similar to those used in proving Theorem 2.1 can then be applied to $L(\alpha, \beta)+\delta L(\alpha, \beta)$, so that

$$
(1+\epsilon)\left\|X_{1} T_{1}(\alpha, \beta)^{-1} Z_{1}\right\|\|\delta L(\alpha, \beta)\| \geq\left\|L(\alpha, \beta)^{-1} \delta L(\alpha, \beta)\right\| \geq 1
$$


and

$$
(1+\epsilon) \kappa_{1}\|\delta L(\alpha, \beta)\| \geq\left\|T_{1}(\alpha, \beta)^{-1}\right\|^{-1}, \quad \kappa_{1} \equiv\left\|X_{1}\right\|\left\|Z_{1}\right\| .
$$

Replacing $\left\|T_{1}(\alpha, \beta)^{-1}\right\|$ by an upper bound (as in Appendix A where $T_{1}$ is in Jordan or Kronecker form) yields results similar to those in Theorem 2.1, but for the cluster in $T_{1}$ rather than the whole spectrum $\sigma(L)$. Here $p$ will be the size of the largest Jordan block associated with the cluster in $T_{1}$. Ignoring higher-order terms in $\epsilon$, the perturbation results will then involve $\kappa_{1}$ instead of $\kappa$. The price to pay for the sharper result is the restriction that the perturbation $\delta L$ has to be small (in the sense of (2.3)), whereas in Theorem 2.1 it can be arbitrary.

(d) In (c) above, when $T_{1}$ contains a simple eigenvalue, $\kappa_{1}$ will be the product of the norms of the corresponding left- and right-eigenvectors. Similarly, for a group of multiple eigenvalues, the corresponding condition number will be the product of the norms of the corresponding left- and right-eigenvectors (or deflating subspaces). Analogous condition numbers can be obtained for clusters of eigenvalues.

(e) Obviously, for large perturbations with $\theta_{1}>1$, we have $\max \left\{\theta_{1}, \theta_{1}^{1 / p}\right\}=\theta_{1}$. On the other hand, when $\theta_{1}<1$, which is usually the case in (c) above, the maximum occurs at $\theta_{1}^{1 / p}$. Furthermore, when the perturbation is asymptotically small, $p$ in (2.1) equals the size of the Jordan block associated with $\left(\alpha_{k}, \beta_{k}\right)$, where the minimum in $s_{(\alpha, \beta)} \equiv \min _{i}\left\{\left|\alpha \beta_{i}-\beta \alpha_{i}\right|\right\}$ occurs at $i=k$. (In fact, a perturbation can be considered "small" when this correct pairing occurs; see the proof in Theorem 3.1.) Notice that the $p$ th root is a common feature in perturbation results for multiple eigenvalues.

(d) A feature of the Bauer-Fike-type perturbation result is that one starts with a perturbed eigenvalue $(\alpha, \beta)$ whose spectral variation from a nearby unperturbed eigenvalue $\left(\alpha_{i}, \beta_{i}\right)$ is bounded. As the size of the perturbation is unrestricted, there may well be unperturbed eigenvalues that are not paired up with any perturbed eigenvalues.

\section{Bauer-Fike theorem for palindromic pencils}

For the pencil $\lambda Z-Z^{\star}$, we can work from the Kronecker canonical form

$$
Q_{1}^{\star}\left(\lambda Z-Z^{\star}\right) Q_{2}=\lambda \Lambda_{+}-\Lambda_{-}, \quad Q_{1}=\left[P_{1}, P_{2}\right], \quad Q_{2}=\left[P_{2}, P_{1}\right],
$$

where

$$
\Lambda_{+}=\left[\begin{array}{cc}
\Lambda & \\
& I
\end{array}\right], \quad \Lambda_{-}=\left[\begin{array}{cc}
I & \\
& \Lambda
\end{array}\right], \quad \Lambda=\operatorname{diag}\left\{J_{1}, \ldots, J_{N}\right\},
$$

with $J_{i}$ being the Jordan block for $\lambda_{i}$ on or inside the unit circle.

We have the following Bauer-Fike perturbation result.

THEOREM 3.1. Consider the palindromic pencil $L \equiv \beta Z-\alpha Z^{\star}$ with the above Kronecker canonical form. Let $\tilde{Z}=Z+\delta Z, \tilde{L} \equiv \beta \tilde{Z}-\alpha \tilde{Z}^{\star},\left(\alpha_{i}, \beta_{i}\right) \in \sigma(L)$ and 
$(\alpha, \beta) \in \sigma(\tilde{L})$, with the scaling $\left|\alpha_{i}\right|^{2}+\left|\beta_{i}\right|^{2}=1=|\alpha|^{2}+|\beta|^{2}$. The spectral variation of $\widetilde{L}$ from $L$ is defined as

$$
s_{L}(\widetilde{L}) \equiv \max _{(\alpha, \beta)}\left\{s_{(\alpha, \beta)}\right\}, \quad s_{(\alpha, \beta)} \equiv \min _{i}\left\{\left|\alpha \beta_{i}-\beta \alpha_{i}\right|\right\} .
$$

Then, for any Hölder norm $\|\cdot\|$,

$$
s_{(\alpha, \beta)} \leq \max \left\{\theta_{2}, \theta_{2}^{1 / p}\right\}, \quad \theta_{2} \equiv c_{2} \kappa_{2}\|\delta Z\|,
$$

where $\kappa_{2}$ is defined as in (3.2), $p$ is the size of the largest Jordan block in $\Lambda$ and $c_{2}=2 \sqrt{2\left(|\alpha|^{2}+|\beta|^{2}\right)}=2 \sqrt{2}$.

Also,

$$
s_{L}(\widetilde{L}) \leq \max \left\{\theta_{2}, \theta_{2}^{1 / p}\right\} .
$$

PROOF. Applying the techniques in [5], we consider the singular matrix

$$
\begin{aligned}
& Q_{1}^{\star}\left[\beta(Z+\delta Z)-\alpha(Z+\delta Z)^{\star}\right] Q_{2} \\
& \quad=\left(\beta \Lambda_{+}-\alpha \Lambda_{-}\right)\left[I+\left(\beta \Lambda_{+}-\alpha \Lambda_{-}\right)^{-1} Q_{1}^{\star}\left(\beta \delta Z-\alpha \delta Z^{\star}\right) Q_{2}\right],
\end{aligned}
$$

which implies that

$$
\kappa_{2}\left\|\left(\beta \Lambda_{+}-\alpha \Lambda_{-}\right)^{-1}\right\|\left\|\beta \delta Z-\alpha \delta Z^{\star}\right\| \geq 1
$$

where

$$
\kappa_{2} \equiv\left\|Q_{1}\right\|\left\|Q_{2}\right\|
$$

From Appendix A, we have the upper bound

$$
\left\|\left(\beta \Lambda_{+}-\alpha \Lambda_{-}\right)^{-1}\right\| \leq c_{0} \max \left\{\left|z_{i}\right|^{-1},\left|z_{i}\right|^{-p_{i}}\right\}
$$

where $z_{i} \equiv \alpha \beta_{i}-\beta \alpha_{i}, p_{i}$ is the size of the Jordan block associated with $\left(\alpha_{i}, \beta_{i}\right)$ and $c_{0} \leq 2$. Substituting this bound into (3.1), we obtain

$$
\kappa_{2} c_{2}\|\delta Z\| \max \left\{\left|z_{i}\right|^{-1},\left|z_{i}\right|^{-p_{i}}\right\} \geq 1 \Rightarrow \min \left\{\left|z_{i}\right|,\left|z_{i}\right|^{p_{i}}\right\} \leq \kappa_{2} c_{2}\|\delta Z\| .
$$

The conclusions of the theorem then follow.

Comments 3.2.

(a) Note that for the 2-norm or the (Frobenius) F-norm,

$$
\theta_{2} \leq \sqrt{2\left(|\alpha|^{2}+|\beta|^{2}\right)} p\|\delta Z\| .
$$

With the scaling $|\alpha|^{2}+|\beta|^{2}=1=\left|\alpha_{i}\right|^{2}+\left|\beta_{i}\right|^{2}, s_{(\alpha, \beta)}$ becomes the chordal metric $[5,11]$ and $\theta_{2} \leq \sqrt{2} p\|\delta Z\|$. Although the F-norm is not a Hölder norm, the corresponding results can be deduced from the 2-norm results.

(b) Comments similar to those labelled (c)-(f) after Theorem 2.1 apply for Theorem 3.1. The proof of the results for clusters of eigenvalues, using the partitioning of an appropriate resolvent, is similar and will not be repeated here. 
(c) Details associated with palindromic "linearizations" can be found in [16]. Obviously, results in this section are applicable to general palindromic pencils which may not be linearizations of matrix polynomials.

\section{Bauer-Fike theorem for anti-triangular form}

From [18], we have the following anti-triangular canonical form for $\star=T$.

THEOREM 4.1. Let $Z-\lambda Z^{\star}$ be a regular $n \times n$ palindromic pencil. There exists $a$ unitary $U \in \mathcal{C}^{n \times n}$ such that $U^{\star} Z U=\left(m_{i j}\right)$ with $m_{i j}=0$ for $i+j \leq n+1$ (that is, $U^{\star} Z U$ is anti-triangular, with zero elements in the upper left corner).

Note that the result for $\star=H$ can easily be obtained by extending the proofs of [18, Lemma 2.2 and Theorem 2.3]. Note also that we are only interested in the case where $Z-\lambda Z^{\star}$ is regular, which does not hold in [18].

The eigenvalues of the palindromic pencil $Z-\lambda Z^{\star}$ are

$$
\frac{m_{1, n}}{m_{n, 1}^{\star}}, \frac{m_{2, n-1}}{m_{n-1,2}^{\star}}, \ldots, \frac{m_{i, n-i+1}}{m_{n-i+1, i}^{\star}}, \ldots, \frac{m_{n-i+1, i}}{m_{i, n-i+1}^{\star}}, \ldots, \frac{m_{n-1,2}}{m_{2, n-1}^{\star}}, \frac{m_{n, 1}}{m_{1, n}^{\star}} .
$$

Note that $n$ will be even when considering a linearization of a palindromic quadratic pencil [16], but the results in this section hold for any $n$.

Let $N$ be the strict lower right triangular part of $U^{\star} Z U$. Reorganize the antitriangular form in Theorem 4.1 into upper triangular form

$$
\mathcal{P}_{n} U^{\star}\left(Z-\lambda Z^{\star}\right) U=\left(D_{1}+N_{1}\right)-\lambda\left(D_{2}+N_{2}\right)
$$

with the order-reversing permutation matrix $\mathcal{P}_{n}=\left[e_{n}, e_{n-1}, \ldots, e_{1}\right]$; here

$$
\begin{gathered}
D_{2}=\operatorname{diag}\left\{m_{1, n}, m_{2, n-1}, \ldots, m_{n-1,2}, m_{n, 1}\right\}, \\
D_{1}=\mathcal{P}_{n} D_{2} \mathcal{P}_{n}=\operatorname{diag}\left\{m_{n, 1}, m_{n-1,2}, \ldots, m_{2, n-1}, m_{1, n}\right\},
\end{gathered}
$$

where $N_{1}=\mathcal{P}_{n} N$ and $N_{2}=\mathcal{P}_{n} N^{\star}$ are strictly upper triangular.

Using the Schur-like form in (4.1), we can prove the following perturbation result for a palindromic pencil.

THEOREM 4.2. Consider the palindromic pencil $L \equiv \beta Z-\alpha Z^{\star}$, with $N$ being the strict lower right triangular part of its anti-triangular canonical form. Let $\tilde{Z}$ $=Z+\delta Z, \tilde{L} \equiv \beta \tilde{Z}-\alpha \tilde{Z}^{\star},\left(\alpha_{i}, \beta_{i}\right) \in \sigma(L)$ and $(\alpha, \beta) \in \sigma(\tilde{L})$. Assume the scaling $|\alpha|^{2}+|\beta|^{2}=1=\left|\alpha_{i}\right|^{2}+\left|\beta_{i}\right|^{2}$. Then, for any Hölder norm $\|\cdot\|$,

$$
s_{(\alpha, \beta)} \leq \sqrt{2} c_{0} \max \left\{\theta_{3}, c_{3} \theta_{3}^{1 / p}\right\}, \quad \theta_{3} \equiv\|\delta Z\|
$$

for some $p \leq n$ and $c_{0} \equiv \min \{2, p\}, c_{3} \equiv \sqrt{2}\|N\|^{1-1 / p}$.

Also,

$$
s_{L}(\widetilde{L}) \leq \sqrt{2} c_{0} \max \left\{\theta_{3}, c_{3} \theta_{3}^{1 / p}\right\} .
$$


PROOF. Consider the singular matrix

$$
\begin{aligned}
\beta(Z+ & \delta Z)-\alpha(Z+\delta Z)^{\star} \\
= & \left(U^{\star}\right)^{-1} \mathcal{P}_{n}\left[\beta\left(D_{1}+N_{1}+\mathcal{P}_{n} U^{\star} \delta Z U\right)-\alpha\left(D_{2}+N_{2}+\mathcal{P}_{n} U^{\star} \delta Z^{\star} U\right)\right] U^{H} \\
= & \left(U^{\star}\right)^{-1} \mathcal{P}_{n}\left[\beta\left(D_{1}+N_{1}\right)-\alpha\left(D_{2}+N_{2}\right)\right] \\
& \times\left\{I+\left[\beta\left(D_{1}+N_{1}\right)-\alpha\left(D_{2}+N_{2}\right)\right]^{-1} \mathcal{P}_{n} U^{\star}\left(\beta \delta Z-\alpha \delta Z^{\star}\right) U\right\} U^{H} .
\end{aligned}
$$

It is easy to see that

$$
\begin{aligned}
& \left\|\left[\beta\left(D_{1}+N_{1}\right)-\alpha\left(D_{2}+N_{2}\right)\right]^{-1}\right\|\left\|\beta \delta Z-\alpha \delta Z^{\star}\right\| \\
& \quad \geq\left\|\left[\beta\left(D_{1}+N_{1}\right)-\alpha\left(D_{2}+N_{2}\right)\right]^{-1} \mathcal{P}_{n} U^{\star}\left(\beta \delta Z-\alpha \delta Z^{\star}\right) U\right\| \geq 1 .
\end{aligned}
$$

Note that $\left[\beta\left(D_{1}+N_{1}\right)-\alpha\left(D_{2}+N_{2}\right)\right]$ is assumed to be nonsingular, otherwise the results in the theorem become trivial.

With $z \equiv \min _{\left(\alpha_{i}, \beta_{i}\right)}\left\|\beta D_{1}-\alpha D_{2}\right\|=\min _{\left(\alpha_{i}, \beta_{i}\right)}\left|\beta \alpha_{i}-\alpha \beta_{i}\right|, \tilde{D} \equiv \beta D_{1}-\alpha D_{2}$ and $\tilde{N} \equiv \beta N_{1}-\alpha N_{2}$, we have

$$
\begin{aligned}
M & \equiv\left[\beta\left(D_{1}+N_{1}\right)-\alpha\left(D_{2}+N_{2}\right)\right]^{-1} \\
& =\left[I-\left(\beta D_{1}-\alpha D_{2}\right)^{-1}\left(\beta N_{1}-\alpha N_{2}\right)\right]^{-1}\left(\beta D_{1}-\alpha D_{2}\right)^{-1}=\left(I-\tilde{D}^{-1} \tilde{N}\right)^{-1} \tilde{D}^{-1} .
\end{aligned}
$$

As $\tilde{D}^{-1} \tilde{N}$ is nilpotent, there exists some $p \leq n$ such that

$$
\left(I-\tilde{D}^{-1} \tilde{N}\right)^{-1}=I+\tilde{D}^{-1} \tilde{N}+\cdots+\left(\tilde{D}^{-1} \tilde{N}\right)^{p-1}
$$

and we obtain

$$
\|M\| \leq\|\tilde{N}\|^{-1} \eta^{-1} \equiv z^{-1}\left(1+\|\tilde{N}\| z^{-1}+\cdots+\|\tilde{N}\|^{p-1} z^{-p+1}\right) .
$$

With $x \equiv\|\tilde{N}\|^{-1} z$, we have the polynomial $P(x) \equiv x^{p}-\eta\left(1+x+\cdots+x^{p-1}\right)=0$, as in Appendix A or [5]. The only positive root $x$ of $P$ satisfies

$$
x \equiv\|\tilde{N}\|^{-1} z \leq c_{0} \max \left\{\eta, \eta^{1 / p}\right\}
$$

As (4.2) and (4.3) imply

$$
\sqrt{2}\|\delta Z\| \geq\left\|\beta \delta Z-\alpha \delta Z^{\star}\right\| \geq\|M\|^{-1} \geq\|\tilde{N}\| \eta
$$

this and the upper bound in (4.3) then lead to

$$
z \leq c_{0}\|\tilde{N}\| \max \left\{\eta, \eta^{1 / p}\right\} \leq \sqrt{2} c_{0} \max \left\{\|\delta Z\|,\|\tilde{N}\|^{1-1 / p}\|\delta Z\|^{1 / p}\right\} .
$$

As $\|\tilde{N}\| \leq \sqrt{2}\|N\|$, the results in the theorem then follow.

With the chosen scaling $|\alpha|^{2}+|\beta|^{2}=1=\left|\alpha_{i}\right|^{2}+\left|\beta_{i}\right|^{2}, s_{(\alpha, \beta)}$ equals the chordal metric. Note also that $p$ is the integer for which $\tilde{N}^{k} \neq 0$ for all $0 \leq k<p$ and $\tilde{N}^{p}=0$. 


\section{Bauer-Fike theorem for semi-Schur anti-triangular form}

A refinement of Theorem 4.2, with a smaller value for $p$, can be proved. We first refine the decomposition in Theorem 4.1.

THEOREM 5.1. Let $Z-\lambda Z^{\star}$ be a regular palindromic pencil. There exist nonsingular $U, V \in \mathcal{C}^{n \times n}$ such that

$$
V^{\star} Z U=\operatorname{anti-diag}\left\{M_{1}, \ldots, M_{r}\right\}, \quad M_{j}=D_{j}+N_{j},
$$

where $M_{j}$ is anti-triangular with anti-diagonal elements in $D_{j}=$ anti-diag $\left\{\lambda_{j}, \ldots, \lambda_{j}\right\}$.

PROOF. The proof is similar to the standard transformation of a Schur decomposition to the corresponding Jordan canonical form. It suffices to show that it is possible to transform the anti-triangular form $\left(\tilde{U}^{\star} Z \tilde{U}, \tilde{U}^{\star} Z^{\star} \tilde{U}\right)$ in Theorem 4.1 to anti-blockdiagonal form, so that

$$
\begin{aligned}
{\left[\begin{array}{ll}
I & 0 \\
P^{\star} & I
\end{array}\right] \tilde{U}^{\star} Z \tilde{U}\left[\begin{array}{ll}
I & Q \\
0 & I
\end{array}\right] } & =\left[\begin{array}{ll}
I & 0 \\
P^{\star} & I
\end{array}\right]\left[\begin{array}{cc}
0 & T_{1} \\
T_{2} & T_{12}
\end{array}\right]\left[\begin{array}{ll}
I & Q \\
0 & I
\end{array}\right] \\
& =\left[\begin{array}{ll}
T_{1} \\
T_{2}
\end{array}\right], \\
{\left[\begin{array}{ll}
I & 0 \\
P^{\star} & I
\end{array}\right] \tilde{U}^{\star} Z^{\star} \tilde{U}\left[\begin{array}{cc}
I & Q \\
0 & I
\end{array}\right] } & =\left[\begin{array}{ll}
I & 0 \\
P^{\star} & I
\end{array}\right]\left[\begin{array}{cc}
0 & T_{2}^{\star} \\
T_{1}^{\star} & T_{12}^{\star}
\end{array}\right]\left[\begin{array}{ll}
I & Q \\
0 & I
\end{array}\right] \\
& =\left[\begin{array}{ll}
T_{2}^{\star} & T_{1}^{\star}
\end{array}\right]
\end{aligned}
$$

when $T_{1}$ and $T_{2}$ have nonintersecting spectra. Multiplying out the above equations produces

$$
\phi(P, Q) \equiv\left(T_{2} Q+P^{\star} T_{1}, T_{1}^{\star} Q+P^{\star} T_{2}^{\star}\right)=-\left(T_{12}, T_{12}^{\star}\right),
$$

which is uniquely solvable [3].

Similar to Theorem 5.1, but with $p$ being bounded by the maximum size of $M_{j}$, we now have the following refined version of Theorem 4.2.

THEOREM 5.2. Consider the palindromic pencil $L \equiv \beta Z-\alpha Z^{\star}$ with its semi-Schur anti-triangular canonical form (5.1). Let $\tilde{Z}=Z+\delta Z, \tilde{L} \equiv \beta \tilde{Z}-\alpha \tilde{Z}^{\star}, \quad\left(\alpha_{i}, \beta_{i}\right)$ $\in \sigma(L)$ and $(\alpha, \beta) \in \sigma(\tilde{L})$. Assume the scaling $|\alpha|^{2}+|\beta|^{2}=1=\left|\alpha_{i}\right|^{2}+\left|\beta_{i}\right|^{2}$. Then, for any Hölder norm $\|\cdot\|$,

$$
s_{(\alpha, \beta)} \leq \sqrt{2} c_{0} \max \left\{\theta_{4}, c_{4} \theta_{4}^{1 / p}\right\}, \quad \theta_{4} \equiv \kappa_{4}\|\delta Z\|
$$

with $\kappa_{4} \equiv\|U\|\|V\|, c_{0} \equiv \min \{2, p\}$ and $c_{4} \equiv \sqrt{2} \max _{j}\left\|N_{j}\right\|^{1-1 / p}$. 
For sufficiently small perturbations, $p$ is the size of the Schur block $M_{j}$ associated with $\left(\alpha_{i}, \beta_{i}\right)$. In general we have $p=p^{*}$, the maximum size of the Schur blocks $M_{j}$.

Also,

$$
s_{L}(\widetilde{L}) \leq \sqrt{2} c_{0} \max \left\{\theta_{4}, c_{4} \theta_{4}^{1 / p^{*}}\right\} .
$$

PROOF. The proof is exactly the same as that for Theorem 4.2, except that the $\|M\|$ in (4.3) now equals the maximum of the norms of its diagonal blocks. The same argument can be followed in a similar fashion as in the proof of Theorem 4.2, using the diagonal block at which the maximum occurs.

When the perturbation is small enough, this maximum (nearly infinite) occurs at the same block associated with $\left(\alpha_{k}, \beta_{k}\right)$ at which $\min _{i}\left\{\left|\alpha \beta_{i}-\beta \alpha_{i}\right|\right\}$ occurs. This gives a sharper perturbation result, with a smaller $p$ which is just the size of the diagonal block associated with $\left(\alpha_{k}, \beta_{k}\right)$.

Comments similar to those labelled (c)-(f) after Theorem 2.1 apply for the above theorem. Like Theorem 3.1, when we consider sufficiently small perturbations there will be a one-to-one correspondence between the original and the perturbed eigenvalues. The above perturbation bounds can be proved for a particular eigenvalue $\left(\alpha_{i}, \beta_{i}\right)$ with the condition number $\kappa_{4}$ replaced by $\left\|U_{j}\right\|\left\|V_{j}\right\|$. In addition, instead of considering one particular eigenvalue, we can consider a group of neighbouring eigenvalues together. This will increase $p$ or the size of the corresponding semi-Schur block $M_{j}$, but will improve the condition of the linear operator $\phi$ in (5.2) as well as $\kappa_{4}$.

\section{Perturbation by differentiation}

The results in this section are quoted from [7].

Without establishing differentiability or the existence of asymptotic expansions (which can be achieved by using the implicit function approach), perturbation results can be obtained via simple differentiation. See [1] for more details of this approach.

For some fixed $z \neq 0$, consider the palindromic eigenvalue problem

$$
P(\lambda, \rho) x(\rho)=0, \quad P(\lambda, \rho) \equiv \lambda(\rho)^{2} A_{1}^{\star}(\rho)+\lambda(\rho) A_{0}(\rho)+A_{1}(\rho)
$$

with the scaling $z^{\star} x(\rho)-1=0$, where $\rho$ is the perturbation parameter, $A_{0}(0)=A_{0}$ and $A_{1}(0)=A_{1}$. We shall use notation $(\cdot)_{\rho}$ and $(\cdot)_{\lambda}$ to denote the corresponding partial derivatives. For a simple eigenvalue $\lambda$, differentiation produces, at $\rho=0$,

$$
\lambda_{\rho}=-\frac{y^{\star} P_{\rho} x}{y^{\star} P_{\lambda} x}=-\frac{y^{\star} P_{\rho} x}{y^{\star}\left(2 \lambda A_{1}^{\star}+A_{0}\right) x}
$$

and

$$
P x_{\rho}=-\left(\lambda_{\rho} P_{\lambda}+P_{\rho}\right) x, \quad z^{\star} x_{\rho}=0 .
$$

Upon choosing $z=y(0)$ (the left-eigenvector corresponding to $\lambda(0)$ ) we obtain, at $\rho=0$,

$$
x_{\rho}=-P^{\dagger}\left(\lambda_{\rho} P_{\lambda}+P_{\rho}\right) x,
$$

where $P^{\dagger}$ denotes the Penrose generalized inverse [11] of $P$. 
The usual conclusions can be drawn: the right-eigenvector $x$ will be rotated through a big angle, even for a small perturbation, when $\left\|P^{\dagger}\right\|$ is large, that is, when the separation between $\lambda$ and the other eigenvalues is fine. This happens, of course, when the assumption of simplicity for the eigenvalue is close to collapsing.

Note that for palindromic eigenvalue problems with $\star=T$, the eigenvalues $\lambda= \pm 1$ may be multiple and nondifferentiable; thus a more sophisticated approach, like the one in [4], is required.

For perturbation results obtained through the application of Sun and Stewart's approach [9, 19] in terms of the implicit function theorem, see [7]. Asymptotic perturbation series for the eigenvalues and the deflating subspaces have been derived.

\section{Conclusions}

Bauer-Fike-type perturbation results for general matrix polynomials, palindromic linearizations and (semi-Schur) anti-triangular canonical forms have been discussed for perturbations of arbitrary size. These perturbation results complement the ones for asymptotic perturbations given in [7]. Consistent results for simple eigenvalues and their corresponding eigenvectors were obtained using simple differentiation. These results indicate, not surprisingly, that the perturbations of an eigenvalue $\lambda$ and its corresponding deflating subspace $\mathcal{S}_{\lambda}$ are proportional, respectively, to the size of the perturbation and the reciprocal of the gap between $\mathcal{S}_{\lambda}$ and other deflating subspaces. Condition numbers are typically proportional to the products of the norms of the leftand right-eigenvectors or deflating subspaces.

\section{Appendix A. Bounding $\left\|\left(\beta \Lambda_{+}-\alpha \Lambda_{-}\right)^{-1}\right\|$}

For any Hölder norm, given

$$
\Lambda_{+}=\left[\begin{array}{cc}
\Lambda & \\
& I
\end{array}\right], \quad \Lambda_{-}=\left[\begin{array}{cc}
I & \\
& \Lambda
\end{array}\right], \quad \Lambda=\operatorname{diag}\left\{J_{1}, \ldots, J_{N}\right\}
$$

with the eigenvalues of $J_{i}$ all on or inside the unit circle, we have

$$
\left\|\left(\beta \Lambda_{+}-\alpha \Lambda_{-}\right)^{-1}\right\|=\max _{i}\left\|M_{i}\right\|,
$$

where $M_{i} \equiv\left(\alpha I-\beta J_{i}\right)^{-1} \in \mathcal{R}^{p_{i} \times p_{i}}$.

Thus it is sufficient to consider the bound for $\left\|M_{i}\right\|$. Let $z_{i} \equiv \alpha \beta_{i}-\beta \alpha_{i}$, with $\alpha_{i}$ and $\beta_{i}$ being diagonal elements of $I$ and $J_{i}$, respectively. (Here, $\alpha_{i}=1$ and $\left|\beta_{i}\right| \leq 1$; the case where $M_{i} \equiv\left(\alpha J_{i}-\beta I\right)^{-1}$ can be treated similarly, and the symmetric notations used here can be adapted easily.) 
We have the Toeplitz matrix

$$
\begin{aligned}
& M_{i} \equiv\left[\begin{array}{ccccc}
z_{i} & -\beta & & & \\
& z_{i} & -\beta & & 0 \\
& & \ddots & \ddots & \\
& 0 & & z_{i} & -\beta \\
& & & & z
\end{array}\right]^{-1} \\
& =\left[\begin{array}{ccccc}
z_{i}^{-1} & \beta z_{i}^{-2} & \beta^{2} z_{i}^{-3} & \cdots & \beta^{p_{i}-1} z_{i}^{-p_{i}} \\
& z_{i}^{-1} & \beta z_{i}^{-2} & \cdots & \beta^{p_{i}-2} z_{i}^{-p_{i}+1} \\
& & z_{i}^{-1} & \ddots & \vdots \\
& 0 & & \ddots & \beta z_{i}^{-2} \\
& & & & z_{i}^{-1}
\end{array}\right] .
\end{aligned}
$$

We then have

$$
\left\|M_{i}\right\| \leq c_{1} \cdot \max \left\{\left|z_{i}\right|^{-1},\left|z_{i}\right|^{-p_{i}}\right\} .
$$

Finally, $p_{i}$ and $\left|z_{i}\right|$ can be replaced by $p \equiv \max p_{i}$ and $z \equiv \min _{i}\left|\alpha \beta_{i}-\beta \alpha_{i}\right|$, respectively, with $c_{1}=p$.

Alternatively, potentially sharper bounds can be obtained, with $c_{1}$ replaced by $c_{0}$. If $M_{i}^{-1}=z_{i}\left[I_{q_{i}}-z_{i}^{-1} N^{(i)}\right]$ for a nilpotent $N^{(i)}$ such that $\left[N^{(i)}\right]^{q_{i}}=0$, then

$$
M_{i}=z_{i}^{-1} \sum_{j=0}^{q_{i}-1} z_{i}^{-1}\left[N^{(i)}\right]^{j}, \quad\left\|M_{i}\right\| \leq \eta^{-1} \equiv\left|z_{i}\right|^{-1} \sum_{j=0}^{q_{i}-1}\left|z_{i}\right|^{-j} .
$$

For simplicity, let $x=\left|z_{i}\right|$ and $m=q_{i}$. The above definition of $\eta$ leads to the polynomial

$$
P_{m}(x) \equiv x^{m}-\eta\left(1+x+\cdots+x^{m-1}\right) .
$$

Descartes' sign rule (La Géométrie 1637) then implies that $P_{m}(x)$ has at most one positive real root. As $P_{m}(0)=-\eta<0$ and $P_{m}(x)>0$ as $x \rightarrow \infty$, any positive number $x^{*}$ for which $P_{m}\left(x^{*}\right)>0$ is an upper bound of the unique real positive root of $P_{m}(x)$. Simple inspection leads to the upper bounds $x^{*}=c_{0} \eta$ when $\eta>1$, and $x^{*}=c_{0} \eta^{1 / m}$ when $\eta \leq 1$, with $c_{0}=\min \{2, m\}$. Consequently, $c_{0}=1$ when $m=1$ (with $x=\eta$ ), and $c_{0}=2$ when $m>1$.

The details are as follows. When $c_{0} \eta>\eta \geq 1$ and $m>1$,

$$
\begin{aligned}
P_{m}\left(c_{0} \eta\right) & =\left(c_{0} \eta\right)^{m}-\eta \frac{\left(c_{0} \eta\right)^{m}-1}{c_{0} \eta-1} \\
& =\frac{c_{0}^{m+1} \eta^{m+1}-c_{0}^{m} \eta^{m}-c_{0}^{m} \eta^{m+1}+\eta}{c_{0} \eta-1} \\
& =\frac{\left(c_{0}^{m} \eta^{m+1}-c_{0}^{m} \eta^{m}\right)+\left(c_{0}^{m+1} \eta^{m+1}-c_{0}^{m} \eta^{m+1}-c_{0}^{m} \eta^{m+1}\right)+\eta}{c_{0} \eta-1} \geq 0,
\end{aligned}
$$


because $c_{0}^{m+1} \eta^{m+1}-c_{0}^{m} \eta^{m+1}-c_{0}^{m} \eta^{m+1}=\left(c_{0}-2\right) c_{0}^{m} \eta^{m+1}=0$. Thus $c_{0} \eta$ is an upper bound of the root $x$ of $P_{m}$ when $\eta \geq 1$.

When $\eta<1$ and $m>1$,

$$
P_{m}\left(c_{0} \eta^{1 / m}\right) \geq c_{0}^{m} \eta-\eta\left(1+c_{0}+\cdots+c_{0}^{m}\right)=c_{0}^{m} \eta-\eta \frac{c_{0}^{m}-1}{c_{0}-1}=\eta>0 .
$$

Thus $c_{0} \eta^{1 / m}$ is an upper bound of the root $x$ of $P_{m}$ when $\eta<1$.

Finally,

$$
\left|z_{i}\right| \leq c_{0} \max \left\{\eta, \eta^{-q_{i}}\right\} \quad \Rightarrow \quad\left\|M_{i}\right\| \leq \eta^{-1} \leq c_{0} \max \left\{\left|z_{i}\right|^{-1},\left|z_{i}\right|^{-q_{i}}\right\},
$$

and the result follows, with $c_{1}$ replaced by the sharper $c_{0}$.

\section{References}

[1] A. L. Andrew, K.-W. E. Chu and E. Lancaster, "Derivatives of eigenvalues and eigenvectors of nonlinear eigenvalue problems", SIAM J. Matrix Anal. Appl. 14 (1993) 903-926.

[2] S. Bora, "Structured eigenvalue condition number and backward error of a class of polynomial eigenvalue problems", Technical Report 417, DFG Research Centre Matheon, Berlin, 2007.

[3] K.-W. E. Chu, "The solution of the matrix equations $A X B-C X D=E$ and $(Y A-D Z, Y C-$ $B Z)=(E, F) "$, Linear Algebra. Appl. 93 (1987) 93-105.

[4] K.-W. E. Chu, "On multiple eigenvalues of matrices depending on several parameters", SIAM J. Numer. Anal. 27 (1990) 1368-1385.

[5] E. K.-W. Chu, "Perturbation of eigenvalues for matrix polynomials via the Bauer-Fike theorems", SIAM J. Matrix Anal. Appl. 25 (2003) 551-573.

[6] E. K.-W. Chu, T.-M. Hwang, W.-W. Lin and C.-T. Wu, "Vibration of fast trains, palindromic eigenvalue problems and structure-preserving doubling algorithms", J. Comput. Appl. Math. 219 (2008) 237-252.

[7] E. K.-W. Chu, W.-W. Lin and C.-S. Wang, "Asymptotic perturbation of palindromic eigenvalue problems", Taiwanese J. Math. to appear. Preprint, National Centre of Theoretical Sciences, National Tsing Hua University, Taiwan, August 2007.

[8] J.-P. Dedieu and F. Tisseur, "Perturbation theory for polynomial eigenvalue problems in homogeneous form", Linear Algebra Appl. 358 (2003) 71-94.

[9] L. Elsner and J. G. Sun, "Perturbation theorems for the generalized eigenvalue problem", Linear Algebra Appl. 48 (1982) 341-357.

[10] I. Gohberg, P. Lancaster and L. Rodman, Matrix polynomials (Academic Press, New York, 1982).

[11] G. H. Golub and C. F. van Loan, Matrix computations, 3rd edn (Johns Hopkins University Press, Baltimore, 1996).

[12] A. Hilliges, "Numerische Lösung von quadratischen Eigenwertproblemen mit Anwendungen in der Schienendynamik", Master Thesis, Technical University Berlin, Germany, July 2004.

[13] A. Hilliges, C. Mehl and V. Mehrmann, On the solution of palindromic eigenvalue problems, Proc. 4th European Congress on Computational Methods in Applied Sciences and Engineering ECCOMAS (The European Community on Computational Methods in Applied Sciences, Jyväskylä, Finland, 2004).

[14] C. F. Ipsen, "Accurate eigenvalues for fast trains", SIAM News 37 (2004).

[15] D. Kressner, M. J. Pelez and J. Moro, Structured Hölder condition numbers for multiple eigenvalues, UMINF report, Department of Computer Sciences, Ume University, Sweden, 2006. (Revised January 2008; available at http://www.sam.math.ethz.ch/ kressner/pubs.php.).

[16] D. S. Mackey, N. Mackey, C. Mehl and V. Mehrmann, "Linearization spaces for matrix polynomials", SIAM J. Matrix Appl. 28 (2006) 971-1004. 
[17] D. S. Mackey, N. Mackey, C. Mehl and V. Mehrmann, "Structured polynomial eigenvalue problems: good vibrations from good linearizations", SIAM J. Matrix Appl. 28 (2006) 1029-1051.

[18] D. S. Mackey, N. Mackey, C. Mehl and V. Mehrmann, "Numerical methods for palindromic eigenvalue problems: computing the anti-triangular Schur form”, Numer. Lin. Alg. Appl. to appear. Technical Report 409, DFG Research Centre Matheon, Berlin, 2007.

[19] G. W. Stewart and J. G. Sun, Matrix perturbation theory (Academic Press, New York, 1990).

[20] F. Tisseur, "Backward error and condition of polynomial eigenvalue problems", Linear Algebra Appl. 309 (2000) 339-361.

[21] F. Tisseur and K. Meerbergen, "A survey of the quadratic eigenvalue problem", SIAM Rev. 43 (2001) 234-286. 